\title{
芳香杂环硫醚类化合物的合成研究
}

\author{
张变香* 庄永强史瑞雪 \\ (山西大学化学化工学院 太原 030006)
}

\begin{abstract}
摘要 近年来芳香杂环硫醚类化合物作为有机合成中间体及药物合成的结构单元，在医药、功能性材料领域被广泛应 用，其合成方法的研究也成为当前科研工作的热点之一. 综述了近几年芳香杂环硫醚类化合物的合成方法，重点介绍 在金属催化下、无金属催化条件下和经由苯炔来制备芳香杂环硫醚的研究进展，并对其部分反应机理做了相应的阐述. 关键词＼cjkstart芳香杂环硫醚; 金属催化剂; 苯炔中间体
\end{abstract}

\section{Synthesis of Aromatic Heterocyclic Sulfide Compounds}

\author{
Zhang, Bianxiang* Kang, Yongqiang Shi, Ruixue \\ (School of Chemistry and Chemical Engineering, Shanxi University, Taiyuan 030006)
}

\begin{abstract}
Recently, the aromatic heterocyclic sulfide was widely used in medical and functional materials as an intermediate in organic chemistry and a structural unit in drug synthesis. Their synthetic method has become one of the hot research areas. This review summarizes the recent synthetic methods of aromatic heterocyclic thioether, which mainly involves metallic catalyzed and no metal-catalyzed methods, as well as benzene alkyne intermediate method. Some of their synthetic mechanisms were illustrated.
\end{abstract}

Keywords aromatic heterocyclic sulfide; metal catalysis; benzene intermediate

芳香杂环硫醚作为人们熟知的含硫化合物，是一类 重要的有机化合物和有机合成中间体, 在医药、农业、 染料工业和材料等领域都有着广泛的应用价值, 成为当 前研究的焦点和热点之一 ${ }^{[1]}$. 例如: 芳基硫代吲哚 (Arylthioindole)是一种新型微管蛋白的抑制剂; 头孢唑 林(Cefazolin)对葡萄球菌、肺炎球菌、大肠杆菌等具有 较强的作用; 奥美拉唑硫醚(Ufiprzole)是用来合成治疗 胃食管反流性疾病药物的重要中间体. 同时, 通过氧化 作用合成具有手性的亚砜类化合物芳硫醚被发现具有 很好的药物活性 ${ }^{[2]}$ 和工业用途 ${ }^{[3]}$. 例如: 含有氮杂环的 手性亚砜化合物一般具有药物活性和生物活性 ${ }^{\left[{ }^{[b]}\right]}$. OPC-29030 是很好的血小板粘附剂; ZD3638 是一种抗 精神病药物. 基于含杂环芳香硫醚类化合物的重要用途 和使用价值, 近年来吸引了科学家的关注, 并对其合成 方法的研发取得了一次又一次的突破. 利用过渡金属催 化进行交叉偶联反应合成硫醚的方法在之前已有综 述 ${ }^{[4]}$, 本文将在着重总结介绍近年在金属催化剂 $(\mathrm{Pd}$, $\mathrm{Cu}, \mathrm{Ni}$ 等)存在下、无催化剂的条件下以及通过苯㶧中间
体三个方面合成芳香杂环硫醚化合物的研究报道.<smiles>[R]c1ccc2[nH]cc(Sc3cc([R])c([R])c([Y])c3[R])c2c1</smiles><smiles>COc1ccc2[nH]c(SCc3ncc(C)c(OC)c3C)nc2c1</smiles>

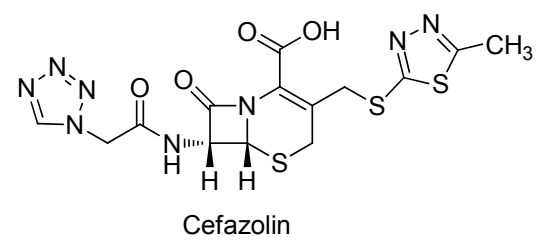<smiles>COc1cccc(-n2ccnc2S(C)(C)O)c1</smiles><smiles></smiles>

ZD3638

\footnotetext{
*E-mail: zbxthh@sxu.edu.cn

Received February 22, 2016; revised March 25, 2016; published online April 15, 2016.

Project supported by the Science and Technology Innovation Project of Shanxi Province (No. 2014101011).

山西省科技创新(No. 2014101011)资助项目.
} 


\section{1 在金属催化剂存在下芳香杂环硫醚的合成}

\section{1 钯催化下的芳香杂环硫醚的合成}

最早的研究集中在含吲哚杂环的芳香硫醚类的合 成. 1978 年 Kosugi 等 ${ }^{[5]}$ 首次在钯催化下将碘苯或溴苯活 化后与硫醇反应，生成了芳基硫醚化合物(Eq. 1). 因该 方法受到反应底物的限制且产率较低, Migita 等 ${ }^{[6]}$ 对反 应进行了改进, 用硫锂试剂代替硫醇与乙烯基溴反应, 得到了较高产率和高立体选择性的芳硫醚类化合物(Eq. 2).

$$
\begin{aligned}
& \mathrm{ArBr}+\mathrm{RSH} \underset{\mathrm{DMSO}}{\stackrel{\mathrm{Pd}(0)}{\longrightarrow}} \mathrm{ArSR} \\
& \stackrel{\mathrm{Ph}}{\underset{\mathrm{Br}}{\xi_{3}}+\mathrm{PhSLi}} \frac{\mathrm{Pd}\left(\mathrm{PPh}_{3}\right)_{4}}{\text { benzene }} \stackrel{\mathrm{Ph}}{\longrightarrow} \underset{\mathrm{SPh}}{\longrightarrow} \\
& \text { 95\% }
\end{aligned}
$$

在以上研究基础上, 1991 年 Dickens 等 ${ }^{[7]}$ 利用吲哚 衍生物的碘取代物, 在 $\operatorname{Pd}\left(\mathrm{PPh}_{3}\right)_{4}$ 催化下与三甲基锡硫 醚化合物反应, 生成了吲哚类的芳香杂环硫醚化合物 (Eq. 3), 产物的收率达到 $89 \%$.

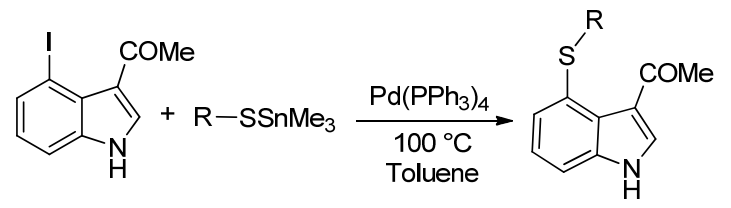

另外, 2009 年 Guo 等 ${ }^{[8]}$ 报道了一种在氯化钯催化下 利用 2-(1-炔基)苯胺和二芳基二硫醚的关环反应生成含 吲哚杂环硫醚的合成法(Eq. 4), 该方法突破了通过吲哚 和硫醇、二硫醚、氨基硫氰酸盐直接反应制备吲哚硫醚 衍生物的传统合成法 ${ }^{[9]}$. 反应体系中原料易得, 反应的 温度较温和且在空气环境中就能够反应.<smiles>[R]C#Cc1ccc[R1]([H])c1N</smiles><smiles>[R]c1[nH]c2ccccc2c1S[Al]</smiles>

与此同时, Chen 等 ${ }^{[10]}$ 利用 2-碘芳胺、端基炔化合物 与芳基硫卤代物在多种催化剂作用下一锅两步法合成 了 3-颈基吲哚衍生物(Scheme 1), 并对其反应机理进行 了探究.

其反应机理如 Scheme 2 所示, 首先在钯一铜催化体 系作用下 2-碘芳基胺与端基炔反应生成中间体 $\mathbf{I}$ 之后, 与芳基硫卤代物反应生成硫鎓盐中间体 II，此中间体因 体系中卤离子的不同得到了不同的产物. 在氯离子作用 下，中间体 II 经过路线 a 快速生成烯硫醚化合物; 同时, 中间体 II 还可以通过路线 $\mathrm{b}$ 关环生成中间体 III, 中间

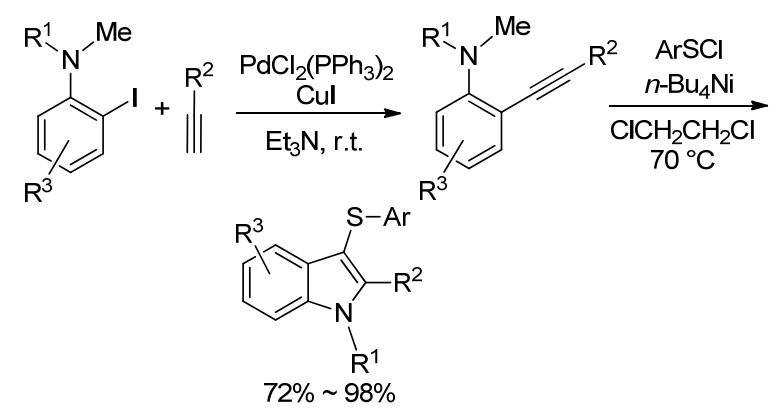

图式 13 -颈基吲哚衍生物的合成

Scheme 1 Synthesis of 3-sulfenylindoles

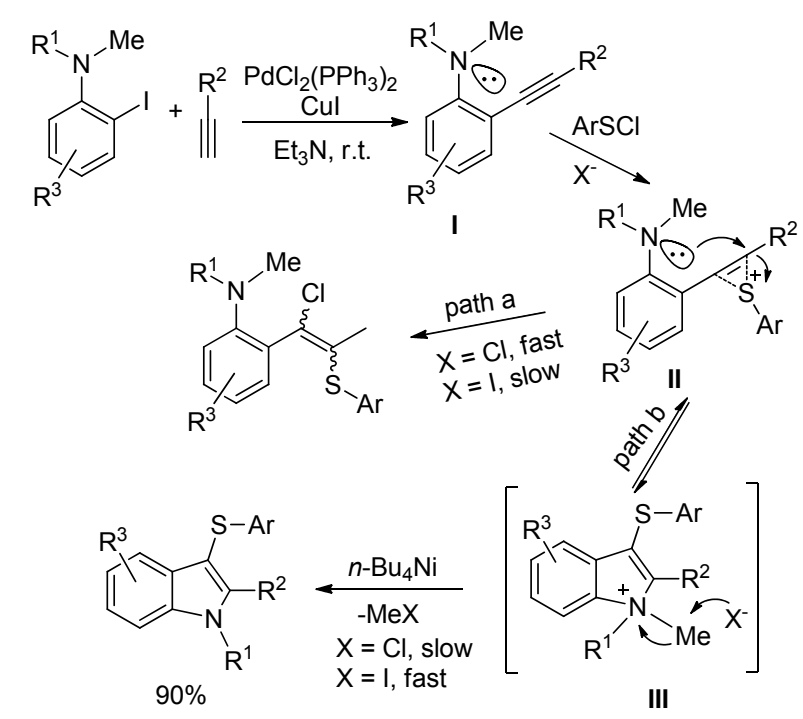

图式 2 2-碘芳胺、端基炔化合物与芳基硫卤代物反应机理 Scheme 2 Mechanism of $N, N$-dialkyl-ortho-iodoanilines and terminal alkynes and arylsulfenyl chlorides

体 III 在碘离子作用下可通过 $\mathrm{S}_{\mathrm{N}} 2$ 反应迅速消去碘甲烷 生成芳香杂环硫醚化合物. 因此可以通过添加碘离子使 反应主要生成含吲哚杂环的硫醚化合物; 也可添加氯离 子使反应主要向生成烯硫醚化合物的方向进行. 该方法 在温和条件下利用简单易得的原料, 通过一锅两步法得 到产率达到 $90 \%$ 以上的产物.

2011 年 Anbarasan 等 ${ }^{[11]}$ 利用较稳定的 $\mathrm{Pd}(\mathrm{OAc})_{2}$ 为 催化剂, 用苯磺酰氧代替苯的氰化物, 在室温下以三氟 乙酸为溶剂，与 9,9-二甲基氧化蒽反应得到了芳杂环硫 醚化合物(Eq. 5). 该方法反应条件温和、避免了其他方 法存在的反应条件苛刻、选择性低等缺点.

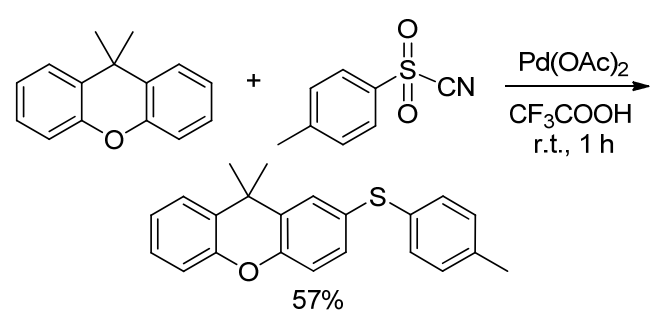


随着研究的不断深入, Cong 等 ${ }^{[12]}$ 在 2013 年和 2014 年分别报道了在钯催化剂和混合配体作用下, 使得杂环 卤代物和硫醇发生 $\mathrm{C}-\mathrm{S}$ 键的交叉偶联反应生成硫醚化 合物的合成法(Eq. 6). 这个方法对于烷基硫醇和芳基硫 醇都有很好的反应活性且产率较高，能够应用于核苷衍 生物的合成.

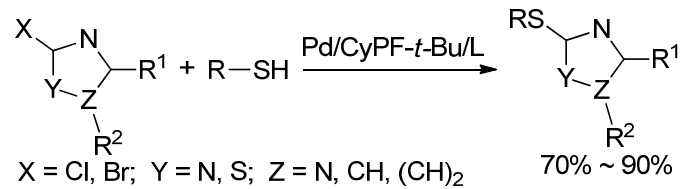

(6)

\section{2 铜催化的芳香杂环硫醚的合成}

近年来, 金属催化的交叉偶联反应已经被证明是一 种非常有效的构建 $\mathrm{C}-\mathrm{S}$ 的方法, 钯是研究和使用最早 最广泛的一种催化剂, 但由于其高昂的价格及对膦配体 的依赖, 使得价格便宜且毒性小的铜催化剂得到了越来 越广泛的研究和应用.

\subsection{1 配体存在下的铜催化}

2004 年, Taniguchi 研究小组 ${ }^{[13]}$ 报道了一种在氧化铜 催化体系中加入镁添加剂来促进二苯二硫醚与卤代芳 烃反应合成产率较高的含氮杂环硫醚化合物的方法(Eq. 7).

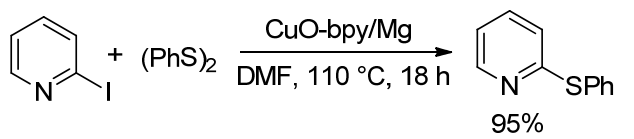

同样利用芳基二硫醚化合物作为硫源，2006 年 Taniguchi 研究小组 ${ }^{[14]}$ 连续进行了碘化亚铜催化下与有 机喼试剂的反应，合成了芳基硫醚化合物(Eq. 8). 随后 对实验进行了改进和扩展, 发现基团的位阻和电子效应 对产率有着较强的影响，取代基 $\mathrm{R}^{1}$ 的给电子效应越强、 位阻越大产物的产率越低.

$$
\mathrm{R}^{1} \mathrm{~S}-\mathrm{SR}^{1}+\mathrm{R}^{2}-\mathrm{B}(\mathrm{OH})_{2} \underset{\substack{\text { Cul-bpy } \\ \mathrm{DMSO} / \mathrm{H}_{2} \mathrm{O}}}{100^{\circ} \mathrm{C}} \mathrm{R}^{1-S} \mathrm{R}^{2}
$$

$\mathrm{R}^{1}=\mathrm{Ph}, \mathrm{R}^{2}=4-\mathrm{MeOC}_{6} \mathrm{H}_{4} 98 \% ; \mathrm{R}^{1}=\mathrm{Ph}, \mathrm{R}^{2}=2-\mathrm{MeOC}_{6} \mathrm{H}_{4} 50 \%$; $\mathrm{R}^{1}=\mathrm{O}_{2} \mathrm{NC}_{6} \mathrm{H}_{4}, \mathrm{R}^{2}=\mathrm{Ph} \quad 97 \% ; \mathrm{R}^{1}=n-\mathrm{Bu}, \mathrm{R}^{2}=\mathrm{Ph} \quad 29 \%$

在此基础上, Luo 等 ${ }^{[15]}$ 提出了无溶剂条件下利用有 机硅与二硫醚化合物反应，可以成功合成硫醚化合物的 方法(Eq. 9). 该反应中所用的有机硅化合物结构稳定、 低毒易得, 是工业化生产的最佳原料; 因反应不需要在 溶剂中进行，既能保护环境又节省了生产成本.

与此同时, Fukuzawa 课题组 ${ }^{[16]}$ 也报道了以碘化亚 铜为催化剂的交叉偶联反应(Eq. 10), 并对其反应机理 进行了阐述, 如 Scheme 3 所示, 首先碘化亚铜与杂环化
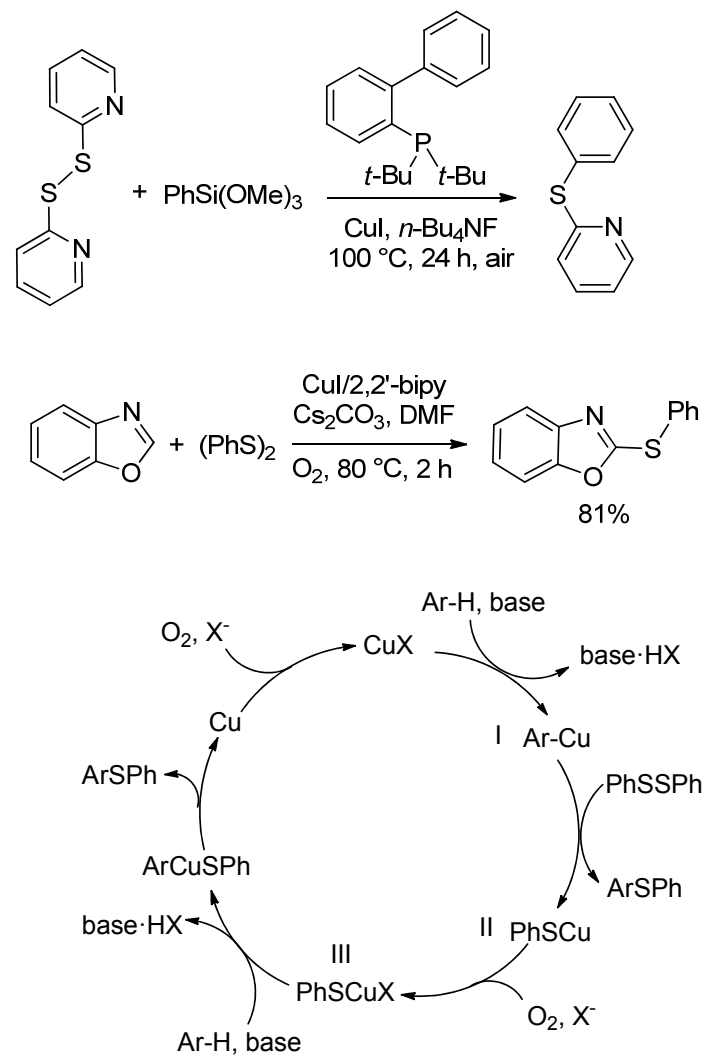

图式 3 芳香杂环化合物与二苯二硫醚的反应机理 Scheme 3 Mechanism of aromatic heterocyclic with diaryl disulfide

合物在碱性条件下反应消去一分子的卤化氢生成中间 体 I. 在中间体 I 的作用下，二硫醚中的 $\mathrm{S}-\mathrm{S}$ 键断裂，生 成一分子的杂环硫醚化合物，同时中间体 II 被氧化生成 中间体 III，紧接着中间体 III 在碱性溶液中与另一分子 的杂环化合物反应生成杂环硫醚和还原态的铜. 还原态 的铜在氧气和卤素离子作用下生成具有催化活性的一 价铜. 此反应体系中二硫醚化合物可以完全转化为芳杂 环硫醚化合物. 但其反应体系局限在芳基硫醇和二芳基 硫醚化合物，不能够与脂肪烃的硫醇反应.

为了使得该反应体系对反应底物有更广泛的适用 范围, 2011 年 Ranjit 等 ${ }^{[17]}$ 对反应进行了改进和完善, 提 出在 2,2-二吡啶、碳酸钠的 $N, N-$ 二甲基甲酰胺(DMF)溶 液中, 氧气环境下苯并噻唑与硫醇反应生成含有杂环的 硫梄化合物的方法(Eq. 11).

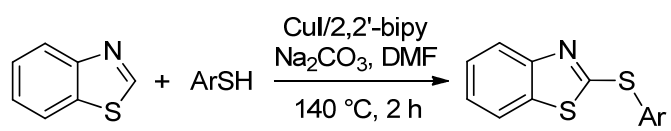

基于路易斯酸对于 $\mathrm{C}-\mathrm{H}$ 键具有很好的活化作用, 2012 年代怊 ${ }^{[18]}$ 以三氟乙酸银为催化剂、 $\mathrm{Cu}(\mathrm{OAc})_{2}$ 为氧 化剂，在氮气保护下使得苯并噻吩等杂环化合物和硫醇 反应得到了含杂环的硫醚(Eq. 12). 同时, $\mathrm{He}$ 等 $^{[19]}$ 在 
2013 年报道了一种以醋酸铜为催化剂、醋酸银为添加 剂, 氧气环境中发生硫醇化的反应(Eq. 13), 其反应机理 与 Scheme 3 类似. 区别在于其使用的氧化剂不同，且反 应体系不需要添加碱.

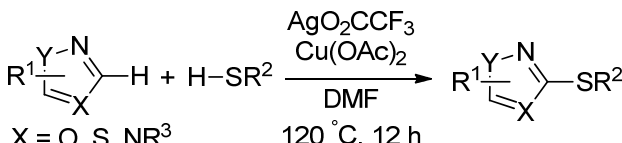

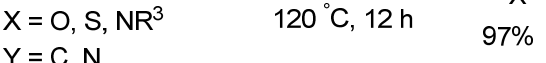

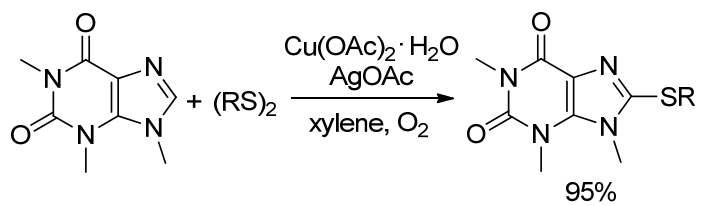

另外，上述反应大多数用 DMF、二甲苯等作溶剂， $\mathrm{Ke}$ 等 ${ }^{[20]}$ 针对溶剂的绿色性提出了一种在水相中利用水 合氯化铜催化剂有效地催化生成 $\mathrm{C}-\mathrm{S}$ 键的方法. 实验 中用 $\mathrm{CuCl}_{2} \cdot 2 \mathrm{H}_{2} \mathrm{O} 、(n-\mathrm{Bu})_{4} \mathrm{NF}$ 、菲、 $\mathrm{Cs}_{2} \mathrm{CO}_{3}$ 等组成催化 反应体系, 使得含杂环的卤化物和作为硫源的 KSCN 反 应生成了产率较好的杂环硫醚化合物(Eq. 14). 该方法 使用水作为反应的溶剂, 是一种对环境友好的合成方 法.

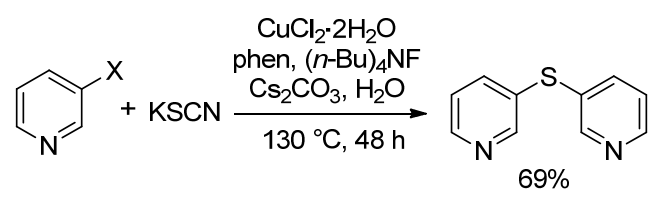

\subsection{2 无配体存在下的铜催化}

针对金属催化反应需要添加昂贵的配体且产物后 处理复杂这一问题, Alves 课题组 ${ }^{[21]}$ 在 2012 年报道了一 种不需要添加剂和配体的铜催化合成方法(Scheme 4). 有趣的是作为硫源的反应底物不同, 反应条件及产率不 同. 当以硫醇作为硫源时, 反应最佳条件为 $5 \mathrm{~mol} \%$ 的催 化剂且需要在氮气保护下进行反应; 当以二芳基二硫醚 作为硫源时, 反应可在空气中进行且催化剂用量可以降 低到 $3 \mathrm{~mol} \%$, 产率达到 90\%以上.

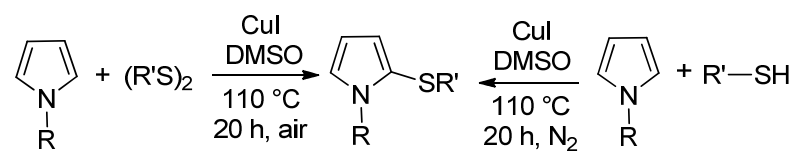

图式 4 铜催化合成吡咯硫醚

Scheme 4 Synthesis of sulfenyl pyrroles by copper-catalyzed

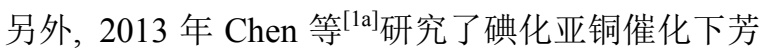
基卤化物和作为硫源的 $\mathrm{S}_{8}$ 作用生成硫醚化合物的反应. 作者指出在这个反应中生成二芳基硫醚和二芳基二硫 醚是一个协同过程, 在氢氧化锂强碱环境中主要生成二
芳基硫醚，在碳酸铯弱碱环境中主要生成二芳基二硫醚 (Scheme 5).

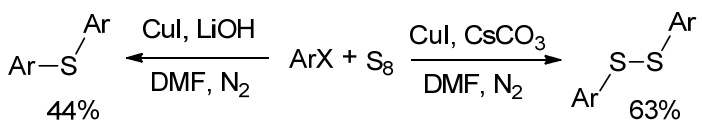

图式 5 用硫磺和芳基碘代物制备芳基硫化物

Scheme 5 S-arylation from sulfur powder and aryl iodide

同年, $\mathrm{He}$ 等 ${ }^{[22]}$ 也报道了一种绿色高效的合成方法， 即以碘化亚铜为催化剂, 叔丁基溴化铵为相转移催化剂 在碱性水溶液中 2-颈基苯并噻唑和芳基卤化物发生交 叉偶联反应，生成了 2-苯硫基苯并噻唑(Eq. 15). 该反应 在 $100{ }^{\circ} \mathrm{C}$ 条件下反应 $24 \mathrm{~h}$ 产率达到 $85 \%$.

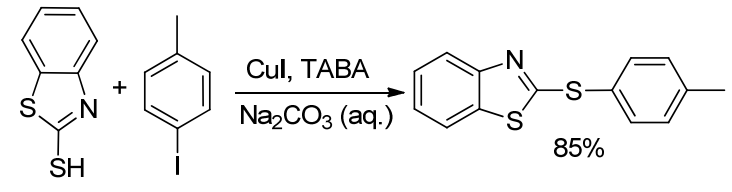

在以上研究的基础上, 2014 年, 本实验室 ${ }^{[23]}$ 将不易 氧化且无难闻气味的二苯二硫醚作为硫源, 在无强碱和 配体的条件下，通过碘化亚铜催化促进 $S-S$ 键断裂后， 与系列苯并五元杂环化合物反应生成了相应的芳基硫 醚. 研究发现催化 $\mathrm{CuI}$ 用量为 $10 \mathrm{~mol} \%$, 溶剂为二甲基 亚砜(DMSO), $110{ }^{\circ} \mathrm{C}$ 温度下反应 $12 \mathrm{~h}$ 时产率达到 $89.9 \%$. 其中吲哚类和苯并噻吩类反应效率较好 (Eq. 16). 其反应机理如 Scheme 6 所示, 首先在碘化亚铜的 作用下，二苯二硫醚的 $\mathrm{S}-\mathrm{S}$ 键断裂生成一分子的苯硫 酚正离子和一分子的 $\mathrm{PhSCu}$ 中间体，然后苯硫酚正离子 进攻苯并五元杂环化合物电子密度较大的 3 位碳原子, 生成一分子的产物，同时体系中两分子的 $\mathrm{PhSCu}$ 中间体 在 DMSO 的作用下形成二苯二硫醚和碘化亚铜, 完成 循环过程. 因此，在反应 $24 \mathrm{~h}$ 之后，反应混合液的 HPLC 分析中还有少量原料二苯二硫醚. 与上述 Scheme 3 相比, 该反应体系是利用硫鎓盐正离子的亲电性进攻 富电子的芳香杂环化合物生成苯硫酚正离子，从而生成 芳香杂环硫醚，而在 Fukuzawa 报道的苯并啞唑与二芳 基二硫醚的反应中需要加入碱、配体及 $101 \mathrm{kPa}$ 氧气. 在 碱的作用下消去氢离子与金属催化剂作用生成碳-金属 键, 反应经过了 $\mathrm{Cu}(\mathrm{I})-\mathrm{Cu}(\mathrm{II})-\mathrm{Cu}(0)-\mathrm{Cu}(\mathrm{I})$ 的催化循环及 $\mathrm{ArCuSPh}$ 中间体的生成.

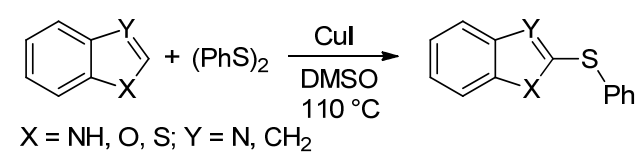

另外, 为了能够得到一种高效低廉的硫化方法以适 应硫化产业的工业化发展, 2015 年姜雪峰等 ${ }^{[24]}$ 创新性地 


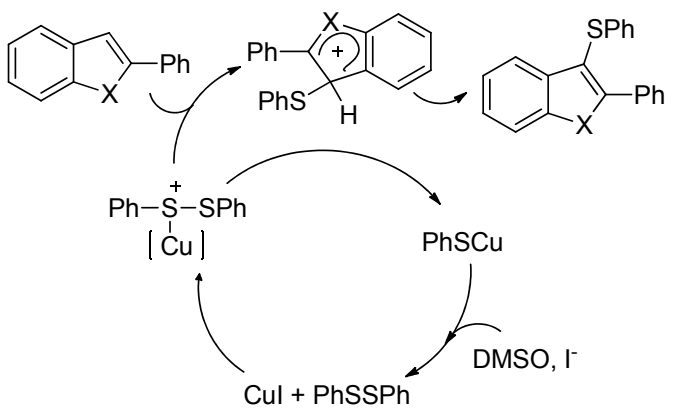

图式 6 苯并杂环化合物与二苯二硫梄的反应机理 Scheme 6 Mechanism of benzo-heterocycle compounds with diaryl disulfide

提出以金属铜为催化剂, 在 DMF 溶剂中使得芳基或者 烯基硫代硫酸盐类衍生物与硼酸、硼酸酯或硼酸盐类衍 生物发生交叉偶联反应, 合成芳基硫醚类化合物的方法 (Eq. 17). 该方法为很多天然产物和药物合成提供了关 键的骨架结构并能够广泛应用于工业化规模的生产.

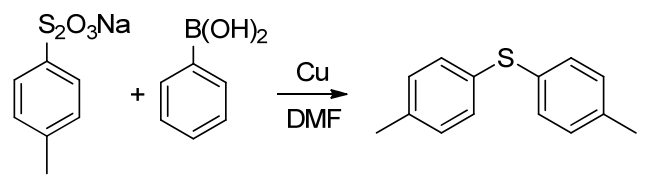

\subsection{3 微波作用下的芳香杂环硫醚合成}

在以上铜催化的合成方法中, 无论有无配体其反应 时间均较长, 针对这一问题, 2013 年 Singh 等 ${ }^{[25]}$ 报道了 以磺酰肼为硫源与芳基卤代物在 $60 \mathrm{~W}$ 微波照射下发生 交叉偶联生成硫醚类化合物的反应(Eq. 18). 该方法使 用微波缩短了反应时间, 1,8 二 二氮杂二环醋酸盐离子溶 液(DBU)稳定性好, 反应活性强.

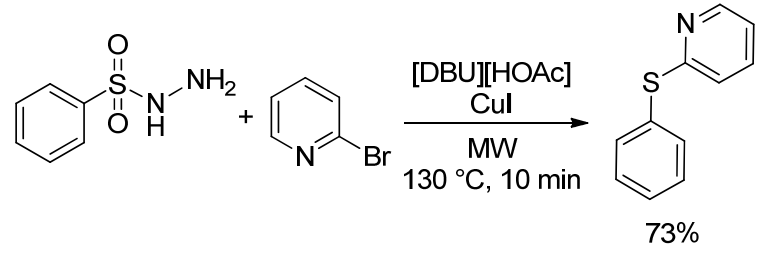

2015 年本实验室 ${ }^{[26]}$ 在微波辐射条件下, 通过 2-颈 基唑类化合物与芳基碘化物的反应，得到了系列芳基硫 醚化合物. 当微波输出功率为 $30 \mathrm{~W}$ 反应时间为 $15 \mathrm{~min}$ 时产率可达到 72\%以上(Eq. 19).

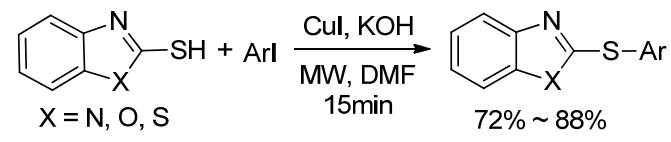

\section{3 镍催化}

与上述钯、铜催化剂相比以镍作为催化剂的反应较 少. Taniguchi ${ }^{[27]}$ 于 2004 年对以往的反应进行了改进, 利
用镍一锌催化体系在中性条件下反应生成了芳基硫梄类 化合物(Eq. 20). 这种方法克服了之前脂肪族的二硫醚 化合物不发生反应的不足 ${ }^{[13]}$.

$$
+(\mathrm{RS})_{2} \frac{\mathrm{NiBr}_{2}-\mathrm{bpy} / \mathrm{Zn}}{\mathrm{DMF}, 110^{\circ} \mathrm{C}, 18 \mathrm{~h}} \underset{60 \% \sim 90 \%}{\longrightarrow-\mathrm{SR}}
$$

为了提高脂肪族硫醚化合物的产率，2006 年, Gomez-Benitez 等 ${ }^{[28]}$ 利用镍的大配体螯合物和锌的催化 体系活化碘苯，与二硫醚反应合成了产率达 $90 \%$ 以上的 芳基硫醚类化合物(Eq. 21). 同时作者对反应机理做了 进一步的研究, 如 Scheme 7 所示, 首先由锌活化镍的大 配体螯合物使其生成具有催化活性的一价镍的配合物, 紧接着与二硫醚化合物配位生成二价镍配合物的颈基 取代物，再与芳基卤化物反应生成一个较大的镍配合 物，经过 $\mathrm{C}-\mathrm{S}$ 键的交叉偶联反应生成芳基硫醚和镍的 大配体螯合物. 从而用配合物的位阻效应解释了该反应 对于脂肪族二硫醚化合物的产率能够达到 $99 \%$ 以上，而 二芳基硫醚化合物的产率却较低的原因(Scheme 7).
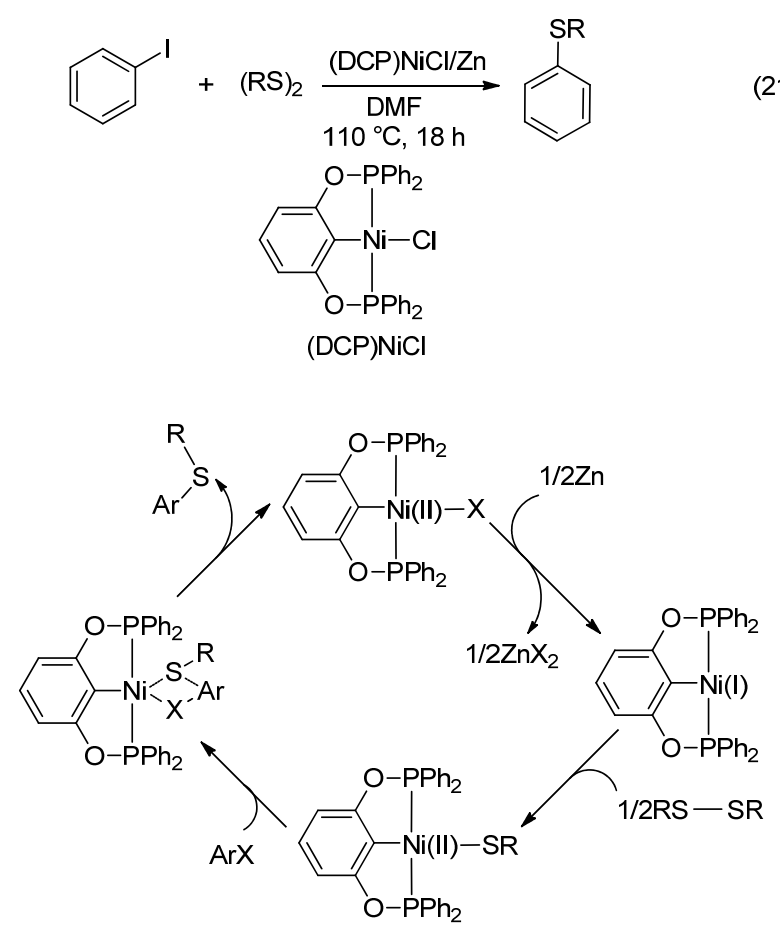

图式 7 碘苯和二硫醚的反应机理

Scheme 7 Mechanism of iodobenzene with disulfide

随着镍配体催化剂在溶剂中的反应研究发展, 2005 年 Gendre 等 ${ }^{[29]}$ 报道了一种利用固相的聚合物作为载体 使得卤代芳烃与硫醇直接发生偶联反应合成芳基硫醚 化合物的方法(Scheme 8). 该方法利用 $N, N$-二异丙基碳 二亚胺(DIC)使碘苯甲酸与载体耦合在一起, 再用硼氢 化物作为催化剂(bpy) ${ }_{2} \mathrm{NiBr}_{2}$ 的载体, 在搅拌条件下使之 
与硫醇盐反应得到硫醚化合物, 用三氟乙酸和二氯甲烷 洗脱得到产物. 产物的后处理和提纯过程操作简单, 杂 质易于出去, 能够有效地合成高纯度产物.

$$
\text { R-SH+ }
$$

图式 8 卤代烃与硫醇直接反应生成硫醚

Scheme 8 The synthesis of thioether by halohydrocarbon and thiol

\section{4 其它催化合成法}

2009 年 Duan 等 ${ }^{[30]}$ 报道了一种利用羧基和颈基在 钯、铜催化体系下生成硫醚化合物反应. 在此基础上, Wang 等 ${ }^{[31]}$ 对反应进行了优化和改进, 在较温和的条件 下以银为催化剂发生脱羧交叉偶联反应得到了芳香杂 环硫醚化合物(Eq. 22), 使得反应温度由 $160{ }^{\circ} \mathrm{C}$ 降低到 了 $60{ }^{\circ} \mathrm{C}$.<smiles>O=C(O)C1COc2ccccc2O1</smiles>

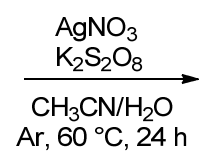<smiles>c1ccc(SC2COc3ccccc3O2)cc1</smiles>

2012 年, $\mathrm{Ge}$ 等 ${ }^{[32]}$ 在温和的条件下以碘为催化剂、 DMSO 为氧化剂使得吲哚衍生物和对称的二硫醚反应 合成了 3-芳硫基吲哚衍生物(Eq. 23); 在此基础上, Azeredo 等 ${ }^{[33]}$ 对反应进行了改进, 在无溶剂微波条件下 高效快速地合成了芳香杂环硫醚化合物(Eq. 24), 是一 种较为绿色的合成法. 2013 年, Yang 等 ${ }^{[34]}$ 以磺酰肼为硫 源与吲哚衍生物反应高效合成了吲哚硫醚化合物(Eq. 25)，其反应条件温和、所使用的溶剂廉价无毒.

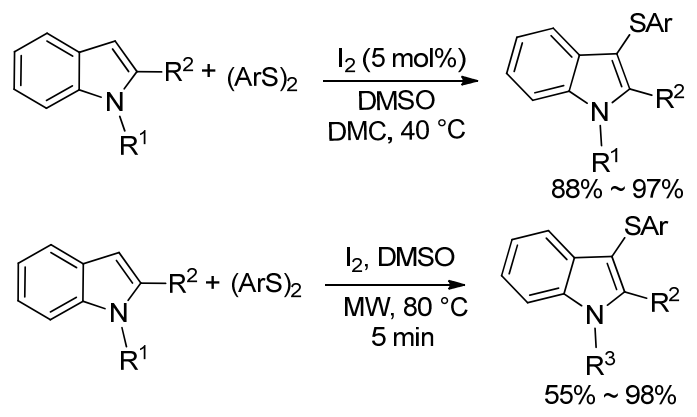

2015 年, Hou 等 ${ }^{[35]}$ 利用一种高选择性地的新型手性 催化剂, 在温和条件下发生三组分迈克尔加成反应, 生

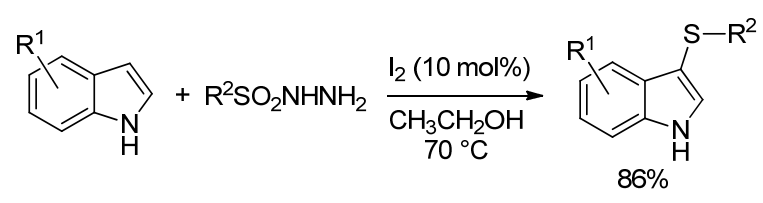

成了具有手性的硫醚化合物(Eq. 26). 反应产物较易转 变为具有生物活性的中间体，在医药生产方面具有很好 地应用前景.

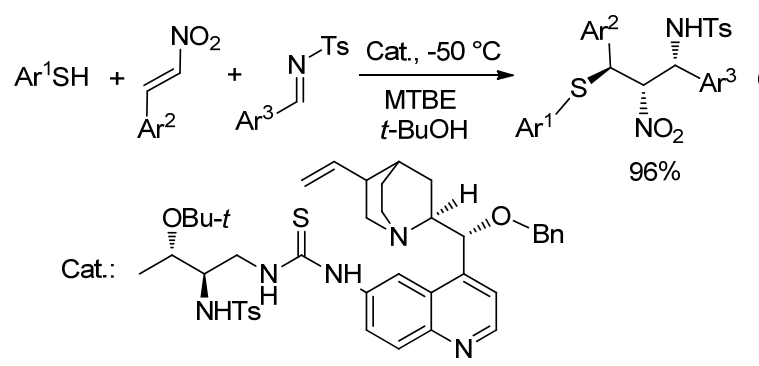

\section{2 无催化剂法}

早期的芳香硫醚是利用卤化物与硫酚钠等试剂反 应来合成的，这种经典的方法反应温度高、时间长且产 率低，限制了在实际生产中的应用; 而过渡金属催化剂 的成本、毒性和对环境的影响等因素严重阻碍了金属催 化的发展、应用和规模化生产. 因此, 在研究金属催化 合成芳香杂环硫醚的同时，科研工作者探究出一些反应 条件温和、对环境友好、原子利用率高的偶联反应来得 到芳香杂环醚化合物的方法.

2003 年 Arguello 等 ${ }^{[1 d]}$ 报道了在光照下合成芳基硫 醚化合物的一锅两步合成法. 首先是芳基卤化物和硫艮 生成芳基硫醇盐，然后与卤代物反应生成芳基硫醚化合 物. 该方法反应条件温和、操作简单且有较好的产率 (Scheme 9).

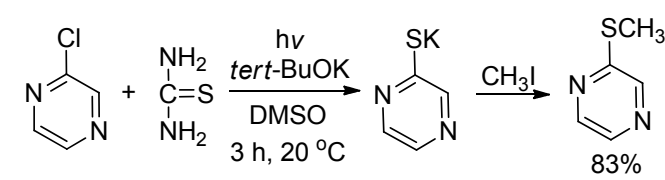

图式 9 一锅两步法合成芳基硫醚化合物

Scheme 9 One-pot two-step synthesis of aryl sulfur compounds

同年, Kitamura 等 ${ }^{[36]}$ 将苯并噻吩盐衍生物在亲和试 剂醇钠溶液中 $50{ }^{\circ} \mathrm{C}$ 下加热反应 $12 \mathrm{~h}$ 后, 生成了产率较 高的硫醚化合物(Eq. 27), 该方法为含有多个芳环的复 杂硫醚类的合成提供了新的思路.

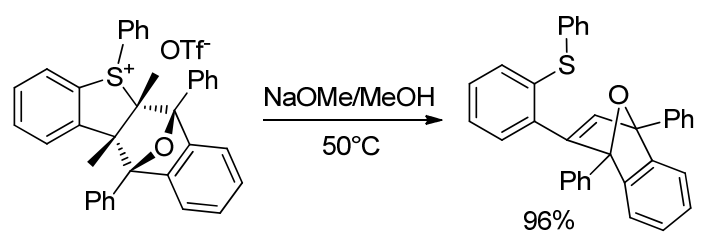


2004 年, Wang 等 ${ }^{[37]}$ 利用二芳基高碘盐与 2-颈基苯 并噻唑，在 1-丁基-3-甲基咪唑的嗍酸盐离子溶液中反 应合成了芳香杂环硫醚化合物(Eq. 28). 该合成方法反 应条件温和, 遗憾的是作者只讨论了 2-颈基苯并噻唑的 反应性

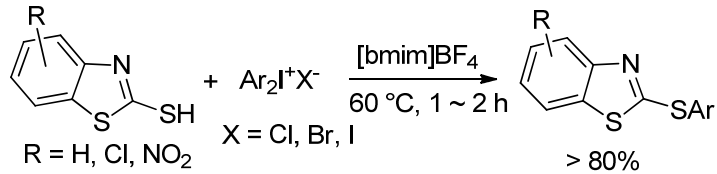

与此同时, Wagner 等 ${ }^{[38]}$ 利用三氟乙酸的协调作用, 将二芳基高碘盐与硫醇反应生成了芳香杂环硫醚化合 物(Eq. 29). 同样，在三氟乙酸的 DMF 溶液中 Zhan 等 ${ }^{[39]}$ 利用微波辐射使得苯并炔醛、2-胺基吡啶和硫醇反应合 成了杂环硫醚化合物(Eq. 30). 这种合成方法得到的杂 环化合物具有很好的生物活性, 可以为医药和天然物质 的合成研究提供重要支架. 同时利用微波辐射缩短了反 应时间、节约了能源和费用, 且较常规方法产物纯净且 产率高.

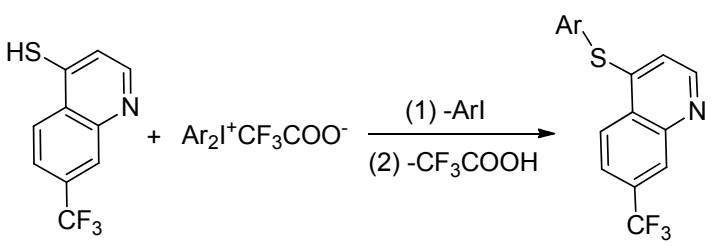

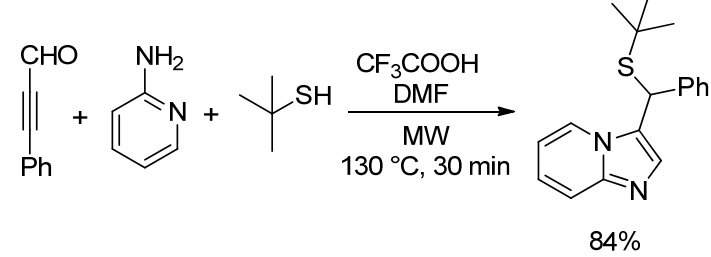

2012 年，周婵等 ${ }^{[2]}$ 报道了利用环硫乙烷的区域选择 性开环制备硫醚的方法(Eq. 31). 由于环硫乙烷在结构 上和环氧乙烷类似, 其键能较环氧乙烷的键能较小, 更 容易发生开环反应; 另一方面环硫乙烷在开环后形成的 颈基和硫负离子具有很好的亲和性, 能够继续反应形成 较复杂的硫醚类化合物.<smiles>COC(=O)C(C)CC(C)C</smiles><smiles>Brc1nc(Br)n(CC2Cn3nc(Br)nc3S2)n1</smiles>

同年，李术艳 ${ }^{[40]}$ 提出在冰醋酸介质中用双氧水氧 化 2-氯吡啶得到较活泼的 2-氯吡啶的氮氧化合物后，可 以与芳基硫酚化合物发生亲电取代反应生成相应的芳 基硫醚类化合物(Scheme 10). 这种合成方法在医药和 农药领域都有着广泛的应用 ${ }^{[41]}$.

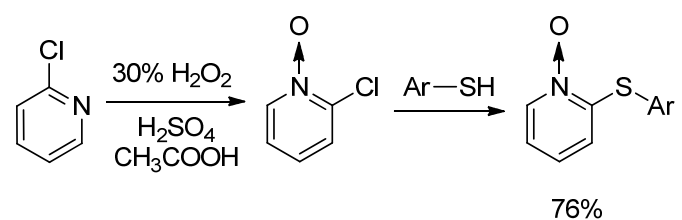

图式 $102-$ 吡啶-N-氧化硫醚的合成

Scheme 10 Synthesis of 2-pyridyl- $N$-oxide thioether

2013 年, Singh 等 ${ }^{[42]}$ 在其研究的基础上利用微波辐 射使得磺酰肼和端基炔反应高效地合成了乙烯基硫醚 类化合物(Eq. 32), 其生成物在材料和医药合成领域有 着广泛的应用价值.

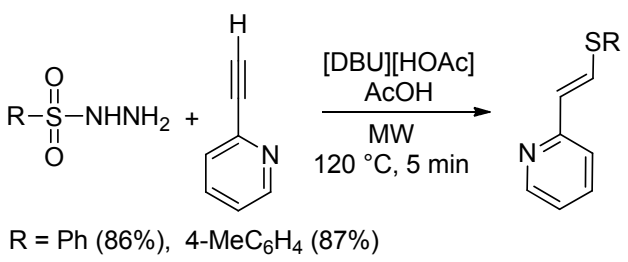

同年，本实验室 ${ }^{[43]}$ 在无金属催化剂、配体及强碱的 条件下，使 2-統基苯并咪唑与二苯基碘三氟甲磺酸盐反 应，高选择性地合成了 $S$-芳基化的 2-苯硫基苯并咪唑 (Eq. 33).

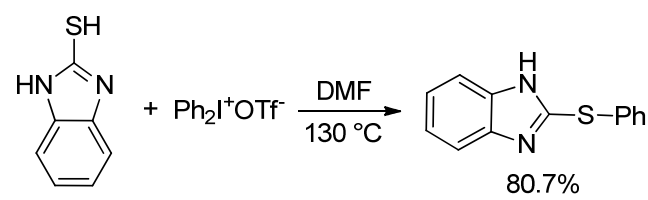

2014 年, Varun 等 ${ }^{[44]}$ 在三氟甲磺酸中将 2-颈基苯并 杂环与芳基化合物发生交叉偶联反应合成了杂环硫醚 化合物. 其中苯环上基团的给电子基效应(如 $\mathrm{OMe}$ )使得 反应高选择性地在其对位发生(Eq. 34).

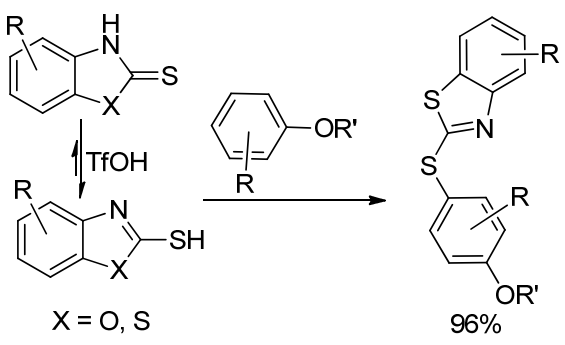

利用苯环上基团的给电子基效应，高兆昶 ${ }^{[45]}$ 用一 
锅法合成了产率为 96\%的 5-溴-3-(3,4,5-三甲氧基苯硫 基)-1H-吲哚(Scheme 11). 实验中只需在 DMF 中加入二 (3,4,5-三甲氧基苯硫基)二硫醚和 NBS 在室温下反应 6 $\mathrm{h}$, 然后再加入 5-溴- $1 H$-吲哚继续反应 $2 \mathrm{~h}$. 这种一锅法 为吲哚类硫醚化合物的合成提供了一种简便高效的方 法. 同年 Yonova 等 ${ }^{[46]}$ 也提出通过一锅法将硫醇、NCS 和有机锌试剂反应能够生成芳基杂环硫醚化合物 (Scheme 12). 该方法反应条件温和, 所用试剂低毒易处 理，可以合成具有生物活性的药物(如艾滋病、乳腺癌、 消炎药物、糖尿病等).

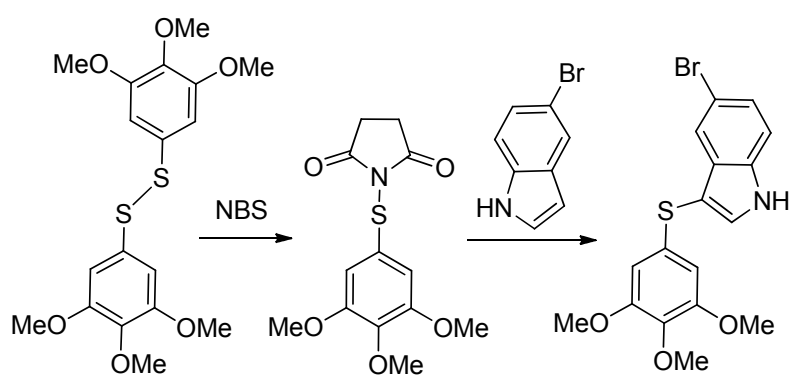

图式 11 5-溴-3-(3,4,5-三甲氧基苯硫基)-1 $H$-吲哚的合成机理 Scheme 11 Synthesis mechanism of 5-bromo-3-[(3,4,5-trimethoxy-phenyl)thio]- $1 H$-indole
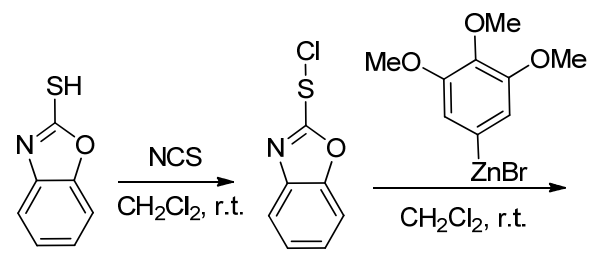

图式 12 一锅法合成芳香杂环硫醚

Scheme 12 One-pot synthesis of heteroaryl thiols sulfide

2015 年, Sun 和 Zeng 等 ${ }^{[47]}$ 提出了以 $\mathrm{K}_{2} \mathrm{~S}$ 为硫源在无 催化剂的条件下与卤代芳烃反应合成硫醚的方法(Eq. 35), 该方法操作简单, 原子利用率高, 且很多的二芳基 硫醚的产率达到了 $96 \%$.

$$
\text { (4h }
$$

\section{3 由苯炔合成芳香杂环硫醚}

苯炔是一类具有高活性的有机反应中间体，作为有 机合成的分子单元被广泛使用. 芳炔中间体有显著的亲 电性且能够同时与亲核性试剂、亲电试剂反应(Scheme 13). 通过该方法可以与硫醇等试剂反应制备硫醚化合 物 $^{[48]}$.

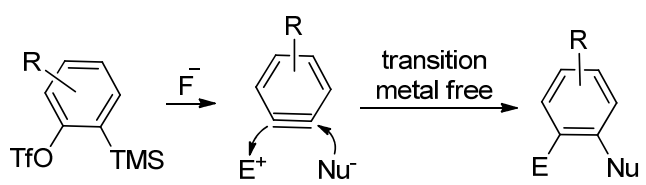

图式 13 苯炔中间体生成硫醚的反应机理

Scheme 13 Synthetic mechanism of sulfide by benzene intermediate

\section{1 无催化剂作用的合成方法}

2005 年, $\mathrm{Lin}$ 等 ${ }^{[49]}$ 用 2 -碘苯二芳基砜与 $i-\mathrm{PrMgCl}$ 在 四氢呋喃中生成苯炔中间体, 然后加入 $\mathrm{RSMgCl}$ 继续反 应 $10 \mathrm{~min}$, 用亲电试剂(如 $\mathrm{I}_{2}, \mathrm{DMF}$, 酰氯等)猝灭反应后 得到了芳基硫醚化合物(Scheme 14). 这种合成方法因 需要在 $-78{ }^{\circ} \mathrm{C}$ 的条件下进行，限制了其在工业上的应 用.

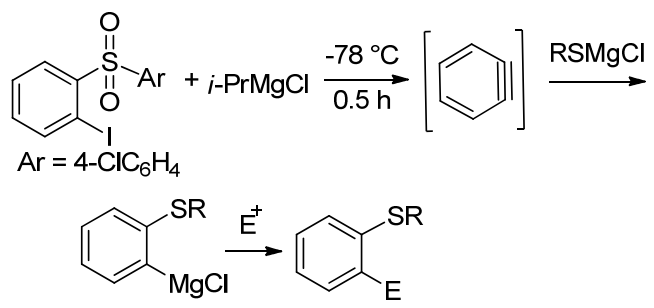

图式 14 通过苯炔的加成反应制备芳硫醚 Scheme 14 Preparation of aryl thioethers by addition reactions to benzyne

2006 年, Zhao 等 ${ }^{[50]}$ 将目前常用的 Kobayashi 前体生 成的芳炔与苯甲酸衍生物发生亲电环化反应生成了芳 香杂环硫醚化合物(Scheme 15). 一般情况下芳炔嵌入 的加成反应中，能够生成稳定的六元环化合物. 因此, 该方法可以用来合成具有药物活性的蒽酮、氧化葱酮和 唂啶酮类化合物及重要的药物合成中间体.

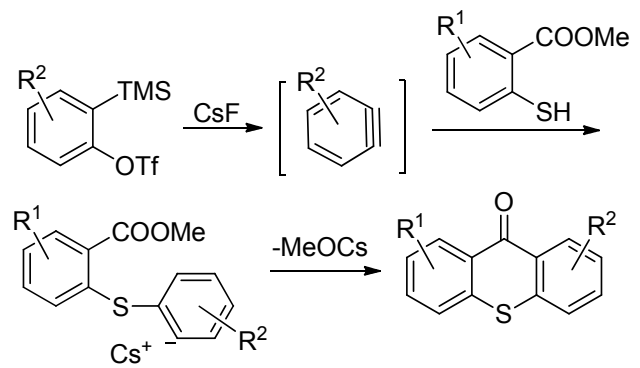

图式 15 通过苯炔中间体合成硫杂葱酮化合物

Scheme 15 Synthesis of thioxanthones by benzene intermediate

2007 年, Toledo 等 ${ }^{[51]}$ 利用 Kobayashi 前体在 CsF 作 用下生成的苯炔, 室温下与二苯二硫醚反应生成了 1,2二苯硫基苯(Eq. 36), 由于反应产物位阻效应较大，反应 $48 \mathrm{~h}$ 产率也仅为 $29 \%$. 


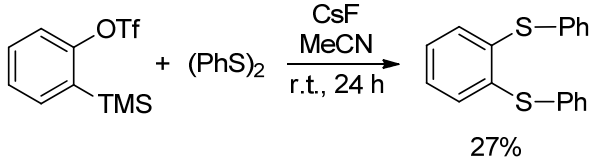

(36)

\section{2 催化剂作用下的合成方法}

2002 年 Edwards 等 ${ }^{[52]}$ 在低温条件下, 利用苯炔一镍 的络合物与含硫化合物 $\mathrm{MeC} \equiv \mathrm{C}-\mathrm{S}-\mathrm{Tol}$ 发生双插入反 应，得到两种同分异构体的硫醚化合物(Eq. 37). 这个合 成法可以构建含䒺骨架的双硫醚类化合物.

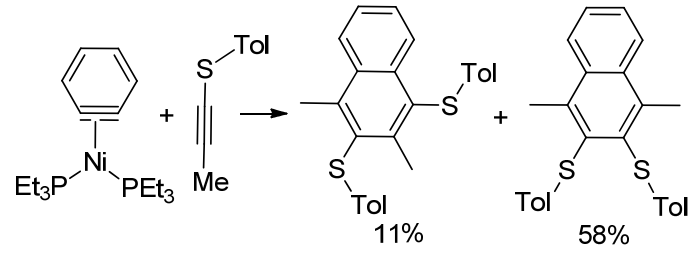

2014 年, Dong 等 ${ }^{[53]}$ 在钯催化下将亲核试剂烯基硫 醚类化合物与苯炔发生 $\mathrm{C}-\mathrm{S}$ 偶联反应, 脱去一分子的 硫醇合成了邻取代的功能化 2-喹啉酮化合物(Eq. 38). 该类化合物在农药、医药的合成领域都有着重要的应用 价值.

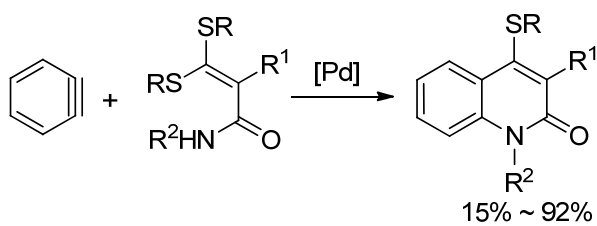

2015 年 Pawliczek 等 ${ }^{[54]}$ 发现利用醋酸钯活化芳基硫 氰酸酯, 在乙腈溶液中与苯炔反应能够得到相应的芳基 硫醚化合物(Eq. 39). 作者指出在反应体系中 $\operatorname{Pd}[0]$ 催化 剂与硫氰酸酯作用形成活化中间体 I, 再与 Kobayeshi 前体生成的苯炔络合成活化中间体 II 之后生成芳香杂 环硫醚化合物和 Pd[0]催化剂(Scheme 16).

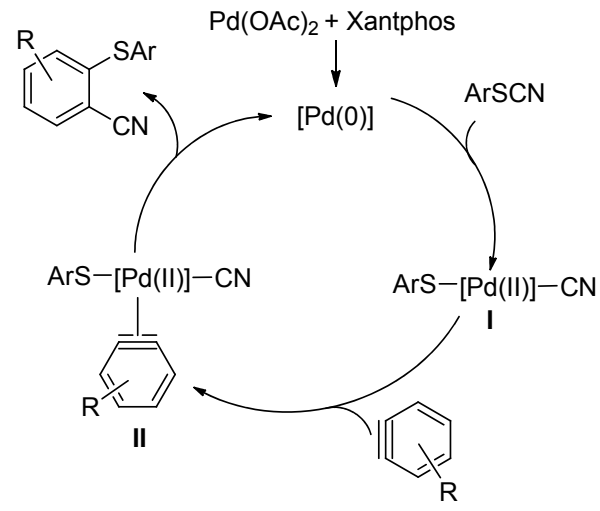

图 16 芳基硫氰酸酯与苯炔的反应机理

Scheme 16 Mechanism of aryl thiocyanates with aryne

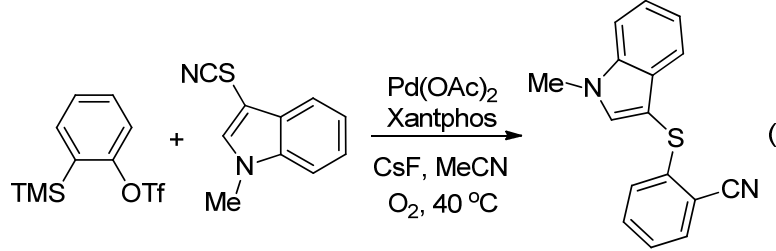

\section{4 结论与展望}

芳香杂环硫醚作为重要的有机化合物和有机合成 中间体，探究其高效的合成法是非常必要的. 利用金属 催化剂的合成技术已经成熟，部分方法产率甚至超过 95\%. 然而这些方法存在反应条件苛刻、操作繁杂、成 本较高且环境污染大的不足，阻碍了金属催化的发展、 应用和规模化的生产。因此，反应条件温和、环境友好 且高效的合成法将不断地蕴育而生. 目前利用芳炔制备 硫醚化合物的报道虽少，鉴于芳炔反应的条件温和、操 作简单、原料易得、反应活性强等优点，芳炔将会成为 医药合成领域和材料领域应用广泛的有机中间体; 并能 够成为大家关注的焦点期待有新的突破.

\section{References}

[1] (a) Chen, H.-Y.; Peng, W.-T.; Lee, Y.-H.; Chang, Y.-L.; Chen, Y.-J.; Lai, Y.-C.; Jheng, N.-Y.; Chen, H.-Y. Organometallics 2013, 32 5514.

(b) Gao, S.; Zeng, Q. L.; Tang, Y. H.; Liu, Y.; Dong, J. Y.; Zhang, B. S. Prog. Chem. 2010, 1760 (in Chinese).

(高珊, 曾庆乐, 唐红艳, 刘洋, 董俊宇, 张斌涁, 化学进展, 2010, 1760.)

(c) Zha, C. J. M.S. Thesis, Hunan University, Changsha, 2011 (in Chinese).

(查长军, 硕士论文, 湖南大学, 长沙, 2011.)

(d) Argüello, J. E.; Schmidt, L. C.; Peñéñory, A. B. Org. Lett. 2003, 5,4133 .

(e) Yao, H.; Richardson, D. E. J. Am. Chem. Soc. 2003, 125, 6211 .

[2] Zhou, C.; Xu, J. X. Prog. Chem. 2012, 24, 338 (in Chinese) (周婵, 许家喜, 化学进展, 2012, 24, 338.)

[3] Yu, S. X.; Liu, H. P.; Fu, C. J. Acta Sci. Nat. Univ. Normal Hunan 2000, 23, 41 (in Chinese).

(俞善信, 刘惠平, 傅长金, 湖南师范大学自然科学学报, 2000 $23,41$.

[4] (a) Beletskaya, I. P.; Ananikov, V. P. Chem. Rev. 2011, 111, 1596.

(b) Shi, S. H.; Tu, X. Z.; Zhang, X. P.; Dai, C.; Zeng, Q. L. Guangzhou Chem. Ind. 2012, 40, 1 (in Chinese)

（史少辉，涂兴钊，张小平，代川，曾庆乐，广东化工，2012，40, 1.)

(c) Tian, F. S.; Wang, Q. Z.; Yang, Z. G.; Fan, X. Y.; Wu, L. J. Zhoukou Normal Univ. 2014, 31, 90 (in Chinese). (田丰收，王全忠，杨志广，凡晓宇，武兰，周口师范学院学报， 2014, 31, 90.)

(d) Zeng, Q. L.; Gao, S.; Allan, K. C. Min-Rev. Org. Chem. 2013, 10, 198

[5] Kosugi, M.; Shimizu, T.; Migita, T. Chem. Lett. 1978, 13.

[6] (a) Migita, T.; Shimizu, T.; Asami, Y.; Shiobara, J.; Kato, Y.; Kosugi, M. Chem. Soc. Jpn. 1980, 53, 1385.

(b) Murahashi, S.; Yamamura, M.; Yanagisawa, K.; Mita, N.; Kon- 
do, K. J. Org. Chem. 1979, 44, 2408.

(c) Foa, M.; Santi, R.; Garavaglia, F. J. Organomet. Chem. 1981, 206, C29.

[7] Dickens, M. J.; Gilday, J. P.; Mowlem, T. J.; Widdowson, D. A. Tetrahedron 1991, 47, 8621.

[8] Guo, Y. J.; Tang, R. Y.; Li, J. H.; Zhong, P.; Zhang, X. G. Adv. Synth. Catal. 2009, 351, 2615.

[9] (a) Wu, G. L.; Liu, Q.; Shen, Y. L.; Wu, W. T.; Wu, L. M. Tetrahedron Lett. 2005, 46, 5831.

(b) Schlosser, K. M.; Krasutsky, A. P.; Hamilton, H. W.; Reed, J. E.; Sexton, K. Org. Lett. 2004, 6, 819.

(c) La Regina, G.; Edler, M. C.; Brancale, A.; Kandil, S.; Coluccia, A.; Piscitelli, F.; Hamel, E.; De Martino, G.; Matesanz, R.; Diaz, J. F.; Scovassi, A. I.; Prosperi, E.; Lavecchia, A.; Novellino, E.; Artico, M.; Silvestri, R. J. Med. Chem. 2007, 50, 2865.

(d) Cacchi, S.; Fabrizi, G. Chem. Rev. 2005, 105, 2873.

(e) Yadav, J. S.; Reddy, B. V. S.; Reddy, Y. J. Tetrahedron Lett. 2007, 48, 7034.

[10] Chen, Y.; Cho, C. H.; Shi, F.; Larock, R. C. J. Org. Chem. 2009, 74, 6802.

[11] Anbarasan, P.; Neumann, H.; Beller, M. Chem. Commun. 2011, 47, 3233.

[12] (a) Cong, M.; Fan, Y.; Raimundo, J.-M.; Xia, Y.; Liu, Y.; Quelever, G.; Qu, F.-Q.; Peng, L. Chem.-Eur. J. 2013, 19, 17267.

(b) Cong, M.; Fan, Y.; Raimundo, J.-M.; Tang, J.; Peng, L. Org. Lett. 2014, 16, 4074.

[13] Taniguchi, N.; Onami, T. J. Org. Chem. 2004, 69, 915.

[14] (a) Taniguchi, N. J. Org. Chem. 2007, 72, 1241.

(b) Taniguchi , N. Synlett. 2006, 1351.

[15] Luo, P.-S.; Yu, M.; Tang, R.-Y.; Zhong, P.; Li, J.-H. Tetrahedron Lett. 2009, 50, 1066.

[16] Fukuzawa, S.-I.; Shimizu, E.; Atsuumi, Y.; Haga, M.; Ogata, K. Tetrahedron Lett. 2009, 50, 2374.

[17] Ranjit, S.; Lee, R.; Heryadi, D.; Shen, C.; Wu, J.; Zhang, P.; Hang, K. W.; Liu, X. J. Org. Chem. 2011, 76, 8999.

[18] Dai, C.; Xu, Z.; Huang, F.; Yu, Z.; Gao, Y.-F. J. Org. Chem. 2012, $77,4414$.

[19] He, Z.; Luo, F.; Li, Y.; Zhu, G. Tetrahedron Lett. 2013, 54, 5907.

[20] Ke, F.; Qu, Y.; Jiang, Z.; Li, Z.; Wu, D.; Zhou, X. Org. Lett. 2011, $13,454$.

[21] Alves, D.; Lara, R. G.; Contreira, M. E.; Radatz, C. S.; Duarte, L. F. B.; Perin, G. Tetrahedron Lett. 2012, 53, 3364.

[22] He, G.; Huang, Y.; Tong, Y.; Zhang, J.; Zhao, D.; Zhou, S.; Han, S. Tetrahedron Lett. 2013, 54, 5318.

[23] (a) Zhang, B. X.; Chen, K.; Yang, L. H.; Xu, Y. T.; Zhang, R. J.; Zhang, L. N.; Shi, R. X. Chin. J. Org. Chem. 2015, 35, 905 (in Chinese).

(张变香, 陈凯, 杨丽花, 许钰涛, 张瑞杰, 张利娜, 史瑞雪, 有 机化学, 2015, 35, 905.)

(b) Zhang, B. X.; Guo, Y. L.; Yang, L. H.; Shi, R. X. Imaging Sci. Photochem. 2015, 33, 177 (in Chinese).

(张变香, 郭一力, 杨丽花, 史瑞雪, 影像科学与光化学, 2015, 33, 177.)

[24] Jiang, X. F.; Qiao, Z. J. CN 104725172A, 2015 [Chem. Abstr. 2015, 163, 191616].
[25] Singh, N.; Singh, R.; Raghuvanshi, D. S.; Singh, K. N. Org. Lett. 2013, 15, 5874.

[26] Zhang, B. X.; Yang, L. H.; Shi, R. X.; Kang, Y. Q. Chin. J. Org. Chem. 2016, 36, 352 (in Chinese).

(张变香, 杨丽花, 史瑞雪, 方永强, 有机化学, 2016, 36, 352.)

[27] Taniguchi, N. J. Org. Chem. 2004, 69, 6904.

[28] Gómez-Benítez, V.; Baldovino-Pantaleón, O.; Herrera-Álvarez, C.; Toscano, R. A.; Morales-Morales, D. Tetrahedron Lett. 2006, 47, 5059.

[29] Gendre, F.; Yang, M.; Diaz, P. Org. Lett. 2005, 7, 2719.

[30] Duan, Z.; Ranjit, S.; Zhang, P.; Liu, X. Chem. Eur. J. 2009, 15, 3666.

[31] Wang, P.-F.; Wang, X.-Q.; Dai, J.-J.; Feng, Y.-S.; Xu, H.-J. Org. Lett. 2014, 16, 4586 .

[32] Ge, W.; Wei, Y. Green Chem. 2012, 14, 2066.

[33] Azeredo, J. B.; Godoi, M.; Martins, G. M.; Silveira, C. C.; Braga, A. L. J. Org. Chem. 2014, 79, 4125.

[34] Yang, F. L.; Tian, S. K. Angew. Chem., Int. Ed. 2013, 52, 4929.

[35] Hou, W.; Wei, Q.; Liu, G.; Chen, J.; Guo, J.; Peng, Y. Org. Lett. 2015, 17, 4870.

[36] Kitamura, T.; Zhang, B.-X.; Fujiwara, Y. J. Org. Chem. 2003, 68, 731.

[37] Wang, F.-Y.; Chen, Z.-C.; Zheng, Q.-G. J. Chem. Res. 2004, 127.

[38] Wagner, A. M.; Sanford, M. S. J. Org. Chem. 2014, 79, 2263.

[39] Zhan, H.; Cao, H.; Qiu, H.; Li, N.; Chen, L.; Liu, J.; Cai, H.; Tan, J. RSC Adv. 2015, 5, 32205.

[40] Li, S. Y. Adv. Fine Petrochem. 2012, 13, 47 (in Chinese). (李术艳, 精细石油化工进展, 2012, 13, 47.)

[41] Shaw, E.; Bernstein, J.; Losee, K.; Lott, W. A. J. Am. Chem. Soc. 1950, 72, 4362.

[42] Singh, R.; Raghuvanshi, D. S.; Singh, K. N. Org. Lett. 2013, 15, 4202.

[43] Zhang, B. X.; Wu, Q.; Chao, J. B.; Guo, Y. L. Chin. J. Synth. Chem. 2013, 21, 86 (in Chinese). (张变香, 吴群, 钞建宾, 郭一力, 合成化学, 2013, 21,86.)

[44] Varun, B. V.; Prabhu, K. R. J. Org. Chem. 2014, 79, 9655.

[45] Gao, Z. C. Chem. Reag. 2013, 35, 1051 (in Chinese). (高兆甫, 化学试剂, 2013, 35, 1051.)

[46] Yonova, I. M.; Osborne, C. A.; Morrissette, N. S.; Jarvo, E. R. J. Org. Chem. 2014, 79, 1947.

[47] Li, Z. J.; Sun, L.X.; Yang, L.; Zeng, Q. L. Fresen. Environ. Bull. 2015, 24, 3686.

[48] Bhunia, A.; Roy, T.; Pachfule, P.; Rajamohanan, P. R.; Biju, A. T. Angew. Chem., Int. Ed. 2013, 52, 10040.

[49] Lin, W.; Sapountzis, I.; Knochel, P. Angew. Chem. 2005, 44, 4258.

[50] Zhao, J.; Larock, R. C. J. Org. Chem. 2007, 72, 583.

[51] Toledo, F. T.; Marques, H.; Comasseto, J. V.; Raminelli, C. Tetrahedron Lett. 2007, 48, 8125.

[52] Edwards, A. J.; Willis, A. C.; Wenger, E. Organometallics 2002, 21, 1654.

[53] Dong, Y.; Liu, B.; Chen, P.; Liu, Q.; Wang, M. Angew. Chem., Int. Ed. 2014, 53, 3442.

[54] Pawliczek, M.; Garve, L. K. B.; Werz, D. B. Org. Lett. 2015, 17, 1716.

(Cheng, F.) 